\title{
Le typage de I'ADN vers l'automatisation des empreintes génétiques
}

$\mathrm{m} / \mathrm{s}$ a suivi la saga des empreintes génétiqucs, initiée en 1985 par Alcc Jeffreys (Leicester, GB), dès ses débuts et l'a régulièrement relatée*. Rappelons que son objectif est d'étudicr la diversité des individus, aussi bien chez l'homme que dans n'importe quelle espèce. Le principe est d'utiliser des zones hypervariables du génome, appelées minisatellites ou VNTR (variable numbers of tandem repeats), qui pcuvent émaner d'un locus unique ou être retrouvés en de nombreux endroits (sondes multilocus)... On peut ainsi affiner de façon précise la distinction entre les individus. L'application la plus évidente portc sur la médecinc légalc, identification d'autcurs de crimes ou de viols, recherche de paternité, mais aussi pour l'évolution, la comparaison d'espèces, ou les mœurs nuptiales d'animaux difficiles à suivre comme les cétacés. Toutefois ces méthodes ont fait l'objet de critiques invoquant des crrcurs dans des procès retentissants $\left(\mathrm{m} / \mathrm{s} n^{\circ} 8\right.$, vol. 5, p. 593). Tout récemment encore, il s'avérait [1] que plusicurs techniciens mis en présence d'un même gcl pouvaient fournir des appréciations différentes. On a donc cherché, pour améliorer les résultats, dans deux directions différentes: l'unc sc borne à perfectionner les techniques fondées sur des différences de taille de séquences avec des sondes multilocus [2, 3] ; l'autre vise à remplacer le modèle purcment quantitatif, fondé sur un nombre variable de répétitions identiques, par un modèle qualitatif.

* Références de in/s concemort les empreintes génétiques:
$n^{\circ} 6$, vol. 1, p. $333 ; n^{\circ} 3$, vol. 2, p. $165 ; n^{\circ} 7$,
vol. 2, p. $401 ; n^{\circ} 6$, vol. 3, p. $370 ; n^{\circ} 7$, vol. 4,
La méthode adoptée par l'équipe de Jeffreys a été schématiséc antéricurement dans $\mathrm{m} / \mathrm{s}$ ( $n^{\circ} 6$, vol. 6, p. 690). Ellc se fonde sur l'hétérogénéité de structure à l'intérieur d'un minisatellite. Lc choix s'est porté sur un locus hypervariable, D1S8, situé sur le chromosome 1. L'unité de répétition, qui porte une séquence de longueur constante de $29 \mathrm{pb}$, comporte ou non une mutation interne ct l'on pcut distingucr, par l'enzyme Hac III, les unités suivant qu'elles portent la mutation (voir $\mathrm{m} / \mathrm{s}, n^{\circ} 8$, vol. 6, p. 691). Le nouveau travail de Jeffreys et al. [4] développc les possibilités de cettc méthode qui, à l'origine, était laboricuse ct cxigeait l'obtention des allèles à l'état haploïde. La méthode a été appclée par scs auteurs MVR-PCR (minisatellite variant repeats avec amplification par PCR). On part des deux allèles dénommés a et $t$ suivant qu'ils portent ou non la mutation; ccs deux allèles diffèrent à leur extrémité 3' qui sert d'amorce différentielle, l'autre amorce provenant d'une séquence flanquante fixc (firure 1). On amplifie ainsi l'un ou l'autrc des allèles pour chaque unité, et on peut en déduire une carte MVR, sous forme d'une échelle lisible selon un code binaire. Dans un petit nombre de cas, un "barreau " de l'échelle ne donne aucune réponse indiquant une variation différente et qui cst codée 0 . Ce code binaire est directement lisible si l'on part d'allèles séparés. Mais le gros progrès de la méthode est que l'on peut partir d'ADN génomique diploïde. Dans ces conditions, on obtient un profil des deux allèles surimposés engendrant un code ternaire pour deux allèles variants (figure 2). En effet, la présence de deux allèles donne le type 1 ; celle de $2 \mathrm{t}$, le type 2 ; celle d'un a et d'un t, le type 3. De plus, la possibilité d'un variant 0 conduit à trois types rarcs : $4(\mathrm{a} 0)$; 5 (t0) ; $6(00)$, ce dernicr donnant un vide sur l'échclle. Le typage MVR-PCR pratiqué sur 334 sujets non apparentés au long de 50 unités répétitives montre une moyenne de 30 misappariements (entre 7 et 50) lorsqu'on compare les individus deux à deux.

Ccttc extraordinaire variabilité ne peut être maintenue qu'à travers un taux élevé de mutations. Il est important de l'évaluer car unc vitcsse de mutation excessive ôterait toutc valeur à la méthode pour la distinction entre les individus. Les autcurs se sont fondés pour ce travail sur les familles bien répertoriées du CEPH de Paris. Sur 286 descendants, sept ont montré des mutations, deux d'entre elles semblant provenir d'une recombinaison interallélique. Ces résultats diffèrent de ccux qui avaient été obtenus auparavant et qui n'étaicnt pas en faveur d'unc recombinaison. L'examen de l'ADN diploïde scmble au contraire montrer que le minisatellite étudié est lc siège d'un point chaud de recombinaison.

Quelles sont les promesses de cette nouvelle méthode? Elle cst, d'abord, bcaucoup plus rapide que la précédente. Mais ses avantages capitaux sont : (a) on peut travailler sur l'ADN génomique total sans avoir à séparer les allèles ; (b) les profils d'ADN sont obtenus sous forme de séquences digitales ressemblant à des codes barres accessibles à l'analysc par ordinateur. Les données sont objectives, chaque expérience portant son témoin interne, et il n'est plus besoin de comparer sur un gel des échantillons côte à côte ; (c) du fait que l'on travaille sur des séquences très courtes, on peut utiliser de l'ADN dégradé, avantage con- 


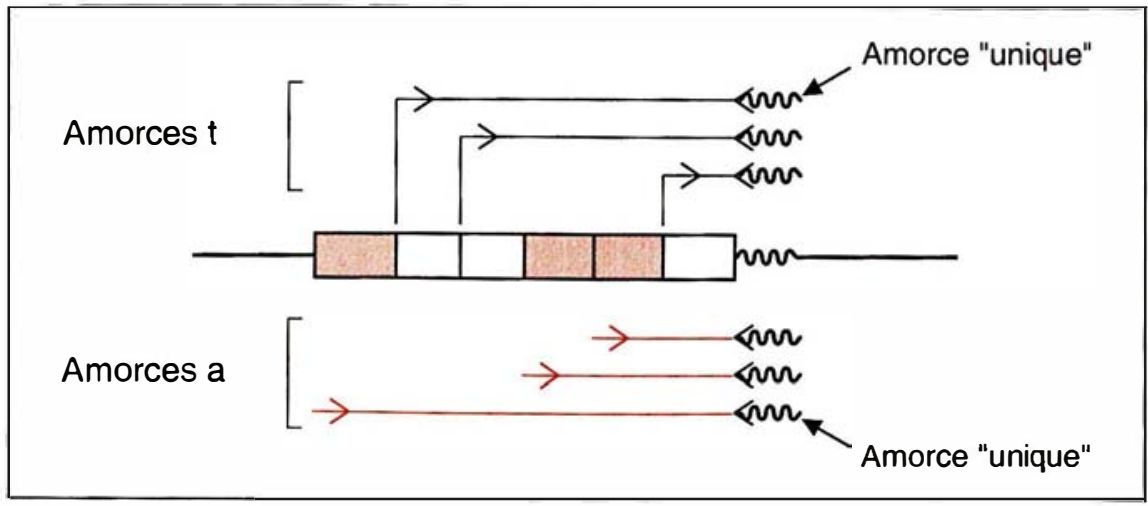

Figure 1. Principe de la MVR-PCR. L'amplification par PCR est réalisée grâce à deux couples d'amorces : un oligonucléotide spécifique d'une séquence unique flanquante (ligne brisée) et l'un des deux oligonucléotides spécifiques de l'allèle a ou $t$ (respectivement flèches rouges et noires). Ces dernières amorces sont allongées depuis l'une quelconque des unités répétées correspondantes a ou $t$, ce qui engendre des fragments de taille différente donnant, après séparation selon la taille par électrophorèse sur gel, une échelle caractéristique de l'alternance a/t.

sidérablc en médecine légale comme en archéologie. Par ailleurs, il cst facile d'établir rapidement des banques de données très étendues.

Du côté des difficultés, il faut d'abord mentionner qu'on ne peut, actuellement du moins, effectuer qu'un petit nombre de cycles de PCR, insuffisant pour révéler les produits par le bromure d'éthidium. Après une amplification trop importante, en effet, les diagrammes tendent à se brouiller, devenant ininterprétables. Plus important, le nombre de minisatellites utilisables est faible: il faut qu'il y ait variation interne, mais pas de variation de la longueur des répétitions, ce qui codage, ertir de phase les échelle part des minisatellites connus. Enfin et interdit les études sur l'evc tes qui n'auront pas ce défaut.

A la veille, semble-t-il, de la commercialisation (Cellmark, une filiale de la multinationale ICI, Imperial Chemical Industries), il faut évoquer, comme le fait Jean-Yves Nau (le Monde) et comme tente d'y répondre Eric Lanton lêne pour le recherch des que l'on trouvera d'autres minisatelli- der de Cambridge (MA, USA) [5], les deux risques majeurs que fait courir la méthode. Le premier est cclui des erreurs possibles ; cclles-ci n'émaneront plus de comparaisons trop subjectives entre des gels d'électrophorèse, mais d'erreurs d'amplification dues à la PCR. Le second est la facilité de mise en fiche progressive d'un nombre croissant d'individus. Il faudra en tenir compte lors de la mise en ouvre de lois sur la bioéthique. L'on ne saurait cependant considérer l'ensemble de ces travaux de manière négative, ni en limiter à la médecine légale les perspectives qui s'ouvrent dans bien d'autres domaines.

Mentionnons, pour finir, l'intérêt que portent les Britanniques au nom de Jeffreys, par une anecdote rapportée par Carr et Goodfellow dans un éditorial de Nalure [6]. Dans l'histoire du droit britannique, le nom de Jeffreys (George Jeffreys, 1645-1689) est associé aux iniquités qu'en tant que Lord Chancelier du roi Jacques II, il déploya lors des séances restées célèbres sous le nom d'Assises Sanglantes de 1685 , à l'encontre des partisans de Monmouth, accusés de complot contre le roi. Il ne paraît que juste qu'un de ses homonymes devienne le symbole d'une meilleure objectivité de la justice.

J.-C. D.

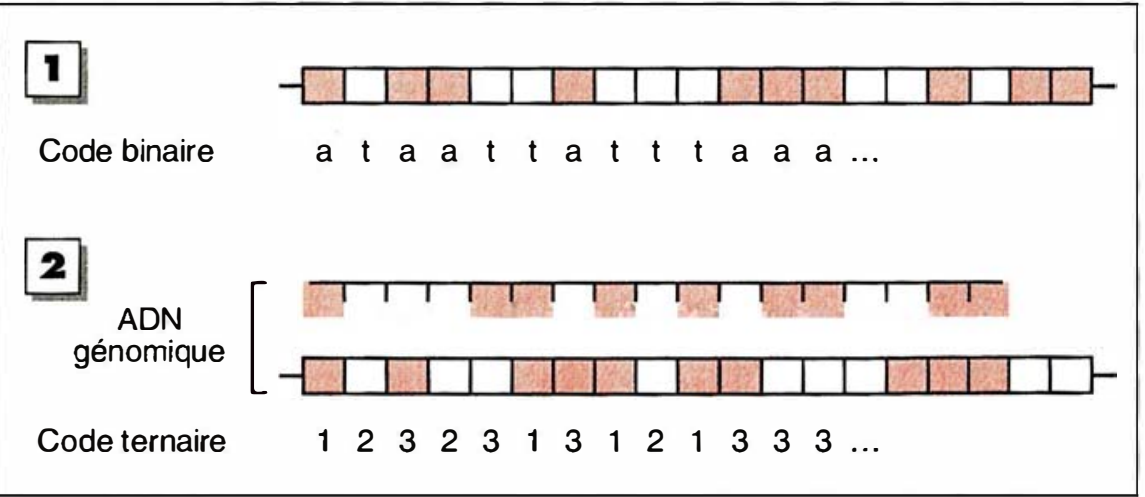

Figure 2. Les principes du codage des unités de répétition d'un minisatellite. Les allèles des unités répétitives se divisent en deux types appelés types a (figuré en rouge) et $t$ (figuré en clair). En (1), sur un allèle isolé, on a une chaîne à code binaire partant de la première unité de répétition. En (2), la surimpression des deux brins donne un code ternaire. On n'a pas représenté les types 4 et 5 (aO et tO), qui donneraient une empreinte moins forte, ni le type 6 (OO), qui n'en donnerait pas du tout. (D'après [4].)

$m / s n^{\circ} 1$, vol. 8 , jonvier 92
1. Shapiro MM, Imprints on DNA fingerprints. Nature $1991 ; 353: 121-2$

2. Jeffreys AJ, Turner M, Debenham P. The efficiency of multilocus DNA fingerprint probes for individualization and establishment of family relationships determined from extensive casework. Am J Hum Genel 1991 ; 48 : 824-40.

3. Budowle B, Giusti AM, Waye JS, et al. Fixed-bin analysis for statistical evaluation of continuous distributions of allelic data from VNTR loci-for use in forensic comparisons. Am J Hum Genet 1991 ; 48 : 841-55.

4. Jeffreys AJ, MacLeod A, Tamaki K, Neil DL, Monckton DG. Minisatellite repeat coding as a digital approach to DNA typing. Nature 1991 ; 354 : 204-9.

5. Lander ES. Invited editorial : research on DNA typing catching up with courtroom application. Am J Hum Genel, 1991 ; 48 : 819-23. 6. Farr CJ, Goodfellow PN. New variations on the theme. Nature $1991 ; 354: 184$. 